\title{
Protective effects of carbon monoxide releasing molecule-2 on pancreatic function in septic mice
}

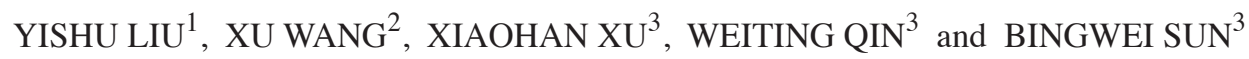 \\ Departments of ${ }^{1}$ General Surgery, ${ }^{2}$ Oncology and ${ }^{3}$ Burns and Plastic Surgery, \\ Affiliated Hospital of Jiangsu University, Zhenjiang, Jiangsu 212001, P.R. China
}

Received May 5, 2018; Accepted December 31, 2018

DOI: $10.3892 / \mathrm{mmr} .2019 .10049$

\begin{abstract}
The present study aimed to investigate the effect of carbon monoxide (CO)-releasing molecule-2 (CORM-2) on pancreatic function in sepsis-model mice. To perform the present investigation, mice were rendered septic by cecal ligation and puncture (CLP). Then, mice were either treated with or without CORM-2 (8 mg/kg, intravenous) for different durations $(6,12$ and $24 \mathrm{~h})$ immediately following CLP. The levels of serum amylase and lipase, tumor necrosis factor $\alpha$, interleukin- $1 \beta$ and interleukin- 6 in addition to myeloperoxidase (MPO) activity in pancreatic tissues were determined at 6,12 and $24 \mathrm{~h}$ post-CLP. Histological scores and the expression of intercellular adhesion molecule 1 (ICAM-1), vascular cell adhesion molecule 1 (VCAM-1), nuclear factor- $\mathrm{KB}(\mathrm{NF}-\kappa \mathrm{B})$ and phosphorylated inhibitor of $\kappa B(p-I \kappa B-\alpha)$ in the pancreas were also evaluated at $24 \mathrm{~h}$ post-CLP. The results of the present study revealed that compared with CLP-alone group, CORM-2 treatment significantly $(\mathrm{P}<0.05)$ reduced the levels of serum amylase, lipase and pro-inflammatory cytokines. In parallel, the severity of pancreatic histology, MPO activity and the expression levels of ICAM-1 and VCAM-1 in the pancreas of CORM-2 treated CLP mice were substantially decreased compared with the untreated group. Furthermore, CORM-2 treatment inhibited the expression levels of NF- $\kappa \mathrm{B}$ and P-IкB- $\alpha$ in the pancreas of mice following CLP compared with the untreated group. CORM-2-liberated $\mathrm{CO}$ exerted protective effects on the pancreatic function of septic mice, and the beneficial effects may be due to the suppression of NF- $\mathrm{kB}$ activation and subsequent regulation of NF-kB-dependent expression of cytokines.
\end{abstract}

\section{Introduction}

Sepsis is considered to be a harmful, non-resolving inflammatory response to infection with the presence of organ

Correspondence to: Dr Bingwei Sun, Department of Burns and Plastic Surgery, Affiliated Hospital of Jiangsu University, 438 Jiefang Road, Zhenjiang, Jiangsu 212001, P.R. China

E-mail: sunliu0423@163.com

Key words: carbon monoxide, sepsis, pancreas, inflammation dysfunction $(1,2)$. In spite of the progress made in the development of medical technology, well-equipped intensive care units and improved practice treatments, the morbidity and mortality rates of sepsis remain high (3). Severe sepsis may cause multiple organ dysfunction (MODS) and the collapse of the circulatory system (septic shock), which mainly results from the extensive activation of inflammatory and coagulation pathways $(3,4)$. Poor clinical outcomes are closely associated with the development of organ dysfunction. The greater the severity of the organ damage, the higher the risk of mortality (5). The dysfunction of organs including the lung, kidney, intestine, liver and brain, in addition to the disorder of including hematopoietic and cardiovascular systems, have been extensively studied (6). However, little attention has been focused on the function of the pancreas in sepsis. Previously, research had identified that the pancreas has a vulnerability to inflammation and injury in patients with sepsis and animal models of sepsis induced by cecal ligation and puncture (CLP) (7).

Carbon monoxide (CO) has long been known as a toxic gaseous molecule due to its ability to combine with hemoglobin. However, a small quantity of endogenous $\mathrm{CO}$, a byproduct of inducible heme oxygenase-1, is continuously generated in mammals (8). Under stress condition, the intracellular levels of CO substantially increase, which has an effect on cyto-protective functions (9). Previously, transition metal carbonyls have been identified as potential CO-releasing molecules (CORMs) with the potential to facilitate the pharmaceutical use of $\mathrm{CO}$ by liberating it to the affected tissues and organs (8). Lipid-soluble metal carbonyl complex tricarbonyldichlororuthenium (II) dimer $\left(\left[\mathrm{Ru}(\mathrm{CO})^{3} \mathrm{Cl}_{2}\right] 2\right)$, known as CORM-2, is the first compound to corroborate the feasibility of this technology, in a controlled manner without substantially altering carboxy-hemoglobin (CO-Hb) levels (10-12). Studies have revealed that CORM-2 is able to modulate inflammation and inhibit the lipopolysaccharide (LPS)-induced production of cytokines in vivo and in vitro (13-15). Previous studies have revealed that CORM-2 attenuates leukocyte sequestration in organs including the lung, liver and small intestine in burned and CLP-induced mouse models of sepsis through interfering with nuclear factor- $\mathrm{\kappa B}(\mathrm{NF}-\mathrm{\kappa B})$ activation and inhibiting the expression of adhesion molecules (16-19). Nevertheless, to the best of our knowledge, no previous studies have determined the regulatory effects of exogenous $\mathrm{CO}$ on pancreatic function in a CLP-induced mouse model of sepsis. 
The present study used a CLP-induced septic mouse model, which was designed as a prospective experiment, to investigate the effects of exogenous $\mathrm{CO}$ on the regulation of pancreatic function and to investigate the molecular mechanisms of underlying the therapeutic effect of CO $(20,21)$. The present study will provide further theoretical foundations and strategies for the treatment of sepsis.

\section{Materials and methods}

Ethics statement. All experiments were performed in accordance with the Guide for the Care and Use of Laboratory Animals published by the US National Institutes of Health (NIH publication no. 85-23, revised 1996; https:/grants.nih.gov/grants/olaw/guide-for-the-care-and-use-oflaboratory-animals.pdf). All experimental protocols were ethically authorized by the Council on Animal Care at Jiangsu University (Jiangsu, China) on the Protection and the Welfare of Animals and conducted in accordance with the National Institutes of Health of China guidelines for the care and use of experimental animals.

Materials. CORM-2, dimethyl sulfoxide (DMSO) and radioimmunoprecipitation assay buffer were purchased from Sigma-Aldrich (Merck KGaA, Darmstadt, Germany). CORM-2 was dissolved in DMSO to acquire a $40 \mathrm{mmol} / \mathrm{l}$ stock solution, as previously described (22). An inactive form of CORM-2 (iCORM-2, used as the negative control) was prepared as followed: The stock of CORM-2 was incubated at $37^{\circ} \mathrm{C}$ in a $5 \% \mathrm{CO}_{2}$ humidified atmosphere for $24 \mathrm{~h}$ to liberate CO. The iCORM-2 solution was finally bubbled with nitrogen to remove the residual $\mathrm{CO}$ present in the solution. The primary antibodies of NF- $\mathrm{NB}$ (sc-7386), phosphorylated inhibitor of $\kappa \mathrm{B}$ (p-IкB- $\alpha$; sc-52943), ICAM-1 (sc-1511) and VCAM-1 (sc1504) were purchased from Santa Cruz Biotechnology, Inc. (Dallas, TX, USA). The nuclear protein extraction buffer kit was obtained from Vazyme (Piscataway, NJ, USA). Other reagents and instruments included tumor necrosis factor- $\alpha$ (TNF- $\alpha$; JER-06) interleukin-6 (IL-6; JEM-04) and IL-1 $\beta$ (JEM-01) enzyme-linked immunosorbent assay (ELISA) kits were all purchased from Joyee Biotechnics Co., Ltd. (Shanghai, China). All other chemicals were of reagent grade and obtained from Sigma-Aldrich (Merck KGaA) unless otherwise stated.

Sepsis mouse model establishment. C57BL/6 mice $(\mathrm{n}=60$; male, 6-8 weeks, weight, $20 \pm 2 \mathrm{~g}$ ) were obtained from the Experimental Animal Center of Jiangsu University, Zhenjiang, Jiangsu, China. Mice were housed in standard wire-topped cages and in temperature-controlled units $\left(18-23^{\circ} \mathrm{C}\right.$ with 40-60\% humidity and 12-hour light/12-hour dark cycle). Food and water were supplied ad libitum. CLP was executed to induce polymicrobial sepsis as previously described $(3,23)$. The mice were anesthetized with $2 \%$ isoflurane in oxygen through a face-mask. Under aseptic conditions, a midline incision $(1-\mathrm{cm})$ was created through the abdominal wall. The cecum was identified then ligated with a 3-0 silk suture at a $1 \mathrm{~cm}$ position from the distal to the base of the cecum. Care was taken not to cause intestinal obstruction. Two punctures of the cecal wall were performed using a 22-gauge needle at the top and bottom. The cecum was lightly squeezed to extrude about $1-\mathrm{mm}^{3}$ droplet of fecal material from the puncture sites to ensure a full-thickness perforation. The cecum was returned to the abdominal cavity, and the incision was closed with 5-0 surgical sutures in layers. Subsequent to the operation, the mice were immediately injected with $1 \mathrm{ml}$ pre-warmed sterile saline subcutaneously to replace the fluid lost. For the sham group animals, mice underwent the same procedure, except with no CLP treatment.

Experimental protocol. A total of 3 time points were selected following a sham operation or CLP treatment in the experiments: 6, 12 and $24 \mathrm{~h}$. At each point in time, a total of 60 mice were randomly divided into 4 groups: Sham group $(n=15)$; CLP group $(n=15)$; CLP+CORM-2 group $(n=15)$; and CLP+iCORM-2 group $(n=15)$. Mice in the CLP+CORM-2 and CLP+iCORM-2 groups received an injection of CORM-2 [8 mg/k intravenously (i.v.)] or iCORM-2 (8 mg/kg, i.v.) immediately subsequent to CLP, respectively. The dosage of CORM-2 used in the present study was based on the results of previous studies $(16,18)$. The negative control (iCORM-2) was used to examine whether the effects observed were due to $\mathrm{CO}$ liberated by CORM-2 or caused by other components of the molecules. Sham animals received normal saline intravenously following the sham operation in the same regimen.

Tissue collection. Mice were euthanized using excessive anesthesia administration and were sacrificed at 6, 12 and $24 \mathrm{~h}$ after CLP or sham surgery as previously described $(23,24)$. Blood samples were collected using cardiac puncture, and the samples were stored in serum tubes and immediately centrifuged at 3,000 x g for $5 \mathrm{~min}$ at room temperature. The serum was isolated from these samples for subsequent lipase and amylase level determination. Pancreatic tissues were removed and immediately frozen in liquid nitrogen or fixed at room temperature overnight in $10 \%$ formalin for further studies.

Biochemical measurement. The activities of serum amylase and lipase were measured to evaluate the pancreatic injury by using a commercial kit (SNM144-BOU, Biolebo, Beijing, China). Levels of amylase and lipase were determined according to the manufacturers' protocol.

Morphological examination. Samples of $10 \%$ formalin-fixed pancreas tissue were embedded in paraffin and sectioned at a thickness of $\sim 4 \mu \mathrm{m}$ for routine histology for each group. Fixed tissues were then stained with hematoxylin and eosin (H\&E) according to the manufacturer's protocol, and examined by two experienced morphologists who were blinded to the sample identity. For these studies, 10 randomly selected microscopic fields (at a magnification of 10x20) were examined for each tissue sample. Each section was scored for the severity of pancreatic injury on a scale of 0-4 (normal to severe) as previously described (25).

Immunohistochemical staining. Pancreatic tissues were fixed in $10 \%$ formalin and $4-\mu \mathrm{m}$ thick sections were prepared from paraffin-embedded tissues. Following deparaffinization and rehydration, and sections were subjected to heat-mediated antigen retrieval in sodium citrate buffer $(10 \mathrm{mM}$ sodium citrate, $\mathrm{pH} 6.0$ ) prior to blocking in $10 \%$ normal goat serum for 
$2 \mathrm{~h}$ at room temperature. The primary antibodies of ICAM-1 and VCAM-1 were diluted to 1/200 and the samples were incubated at $4^{\circ} \mathrm{C}$ overnight. Following three washes with phosphate buffered saline, a horseradish peroxidase (HRP)-conjugated goat anti-rabbit secondary antibody (sc-2004, Santa Cruz Biotechnology, Inc.) was diluted 1:100 and incubated with the samples for $20 \mathrm{~min}$ at room temperature. The slides were then washed again three times with tris-buffered saline. Thereafter, the slides were filled with freshly prepared 3,3'-Diaminobenzidine chromogen (brown) and incubated at room temperature for $3 \mathrm{~min}$, washed in distilled water and counter-stained with hematoxylin for $10 \mathrm{sec}$ at room temperature. This was followed by dehydration with gradient ethanol (100, 95 and $80 \%)$ and clearing using xylene. The slides were then mounted with mounting medium, labelled and viewed under a light microscope. For these studies, 10 randomly selected microscopic fields (magnification, 10x20) were examined for each tissue sample. Mean optical density for ICAM-1 and VCAM-1 were evaluated using Image-Pro Plus version 6.0 (Media Cybernetics, Inc., Rockville, MD, USA).

Myeloperoxidase (MPO) activity.MPOactivity was determined in pancreatic tissues in a procedure similar to that documented in other studies (26-28). The tissue samples were homogenized in $50 \mathrm{mmol} / \mathrm{l}$ potassium phosphate buffer ( $\mathrm{PB} ; \mathrm{pH} 6.0)$ and centrifuged at $10,000 \mathrm{xg}$ for $10 \mathrm{~min}$ at room temperature. The pellets were suspended in $50 \mathrm{mmol} / \mathrm{l} \mathrm{PB}$ including $0.5 \%$ hexadecyltrimethylammonium bromide. Subsequent to sonication, the samples were centrifuged at $10,000 \mathrm{x}$ g for $10 \mathrm{~min}$ again at room temperature. Aliquots $(0.3 \mathrm{ml})$ were added to $2.3 \mathrm{ml}$ of the reaction mixture containing o-dianisidine, $50 \mathrm{mmol} / \mathrm{l} \mathrm{PB}$ and $20 \mathrm{mmol} / 1 \mathrm{H}_{2} \mathrm{O}_{2}$ solution. One unit of enzyme activity was regarded to indicate the quantity of MPO present that resulted in a change in the absorbance measured at $460 \mathrm{~nm}$ for $3 \mathrm{~min}$. MPO activity was expressed as $\mathrm{U} / \mathrm{g}$ tissue.

TNF- $\alpha, I L-6$ and IL-1 $\beta$ level detection. TNF- $\alpha$, IL-6 and IL-1 $\beta$ levels in pancreas tissue homogenates were measured using ELISA kits according to the manufacturer's protocol of each kit.

Western blot analysis. Nucleic proteins were extracted using a nuclear protein extraction buffer kit (Vazyme) according to the manufacturer's protocol. A BCA protein assay kit (Pierce; Thermo Fisher Scientific, Inc., Waltham, MA, USA) was used to evaluate the protein concentrations. Then, samples $(10 \mu \mathrm{g}$ protein) were subjected to electrophoresis on $10 \%$ SDS-PAGE gels using a discontinuous system and transferred onto polyvinylidene fluoride membranes, which were then incubated with an anti-mouse $\mathrm{NF}-\kappa \mathrm{B}$-specific polyclonal antibody (1:1,000; cat no. Sc-514451; Santa Cruz Biotechnology, Inc.), anti-mouse P-IкB- $\alpha$-specific polyclonal antibody $(1: 1,000$; cat no. Sc-7977; Santa Cruz Biotechnology, Inc.) or $\beta$-actin (1:1,000; cat no. Sc-81178; Santa Cruz Biotechnology, Inc.) at $4^{\circ} \mathrm{C}$ overnight and a HRP-conjugated goat anti-mouse immunoglobulin G antibody (1:10,000; cat no. Sc-2031; Santa Cruz Biotechnology, Inc.; incubated at room temperature for $1 \mathrm{~h}$ ) was used as the secondary antibody. Electrochemiluminescence reagent was used to visualize the bands using FluorChem FC3 (ProteinSimple, San Jose, CA, USA), and AlphaView version
3.4.0 software (ProteinSimple, Santa Clara, CA, USA) was used for quantification analysis.

Statistical analysis. Statistical analyses were performed using GraphPad Prism 5 (GraphPad Software, Inc., La Jolla, CA, USA). Data were presented as the mean \pm the standard deviation. One-way factorial analysis of variance followed by a Tukey's post-hoc test were performed for comparisons between the groups. $\mathrm{P}<0.05$ was considered to indicate a statistically significant difference.

\section{Results}

Effect of CORM-2 on the function of the pancreas in septic mice. Sepsis results in MODS, which includes injury of the pancreas $(29,30)$. To demonstrate the protective effects of CORM-2 on the function of pancreas in a CLP-induced mouse sepsis model, the levels of serum amylase and lipase (commonly used as bio-markers to detect pancreatic injury) $(30,31)$ were examined. The level of serum amylase was significantly increased at 6, 12 and 24 h post-CLP compared with the sham mice $(\mathrm{P}<0.05)$ (Fig. 1A). Administration of CORM-2 in septic mice significantly decreased the level of serum amylase at 6 , 12 and 24 h post-CLP compared with the CLP-alone group $(\mathrm{P}<0.05$; Fig. 1A). The septic mice treated with iCORM-2 did not present altered serum amylase activity when compared with the CLP group (Fig. 1A). Similar results were obtained when assessing the serum lipase levels (Fig. 1B).

The damage to the pancreas was also examined by histological evaluation. The pancreatic tissue from the sham group mice exhibited normal architecture, while the pancreatic tissues in the septic mice exhibited severe pathological injury at $24 \mathrm{~h}$ post-CLP (Fig. 2A). The pancreatic tissues in the septic mice exhibited characteristic edema, inflammatory cell infiltration and necrosis of the acinar cells (Fig. 2A). Treatment of the CLP mice with CORM-2 for $24 \mathrm{~h}$ significantly reduced the extent and severity of the histological signs of pancreatic injury compared with the CLP-alone group, including edema, inflammatory cell infiltration and necrosis $(\mathrm{P}<0.05$; Fig. $2 \mathrm{~A}$ and $\mathrm{B}$ ), whereas alleviation in the injury of pancreas was not observed in the iCORM-2 treatment group compared with the CLP-alone group (Fig. 2A and B). The present study revealed that CORM-2 treatment exerted protective effects in the CLP-induced pancreatic damage.

Effect of CORM-2 on MPO activity in the pancreas of septic mice. Inflammatory cell infiltration is a notable pathogenesis of sepsis-induced acute pancreatic injury (32). To evaluate pancreatic neutrophil infiltration, MPO activity, an index of neutrophil accumulation, was measured in the pancreatic tissues obtained from the sham group, the CLP group, the CLP+CORM-2 group and the CLP+iCORM-2 group post-operation at 6,12 and $24 \mathrm{~h}$. The results revealed that MPO activity was significantly increased in septic mice at 6 , 12 and $24 \mathrm{~h}$ post-CLP when compared with the sham group ( $\mathrm{P}<0.05$; Fig. 3). Treatment with CORM-2 subsequent to CLP treatment significantly attenuated MPO activity at 6, 12 and $24 \mathrm{~h}$ compared with the CLP-alone group ( $\mathrm{P}<0.05$; Fig. 3 ), indicating that treatment with CORM-2 decreased the infiltration of neutrophils in the pancreatic tissues. No significant 


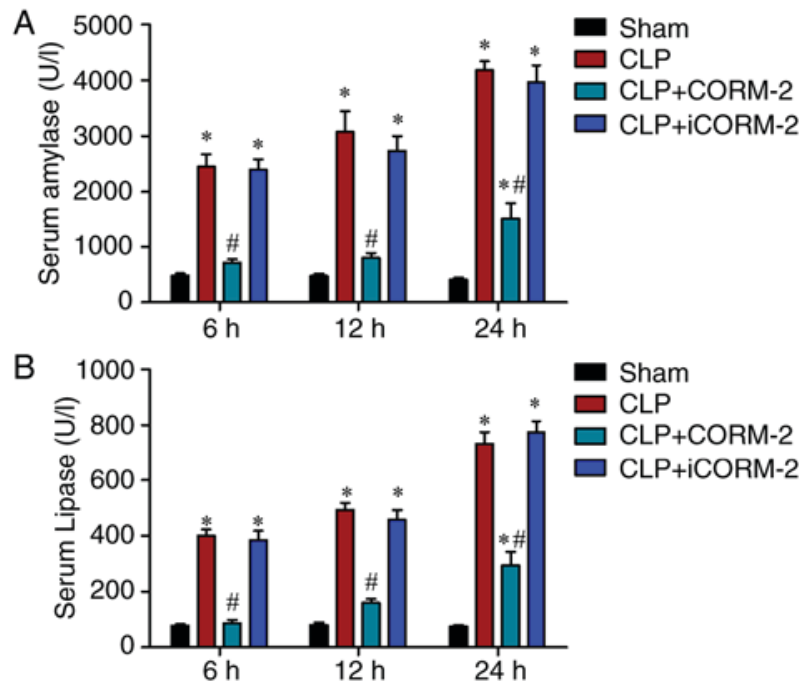

Figure 1. Effect of CORM-2 on serum amylase and lipase levels in CLP-induced septic mice. Serum (A) amylase and (B) lipase levels were determined at 6,12 and $24 \mathrm{~h}$ after the induction of CLP treatment or a sham operation in four groups: A sham group; CLP, mice subjected to CLP to induce polymicrobial sepsis; CLP+CORM-2, mice subjected to CLP and treated with CORM-2 (8 mg/kg, i.v.); and CLP+iCORM-2, mice subjected to CLP and treated with iCORM-2 ( $8 \mathrm{mg} / \mathrm{kg}$, i.v.). ${ }^{*} \mathrm{P}<0.05$ vs. the sham group; ${ }^{\#} \mathrm{P}<0.05$ vs. the CLP group. These data are expressed as the mean \pm standard deviation, $\mathrm{n}=5$ for each group. CLP, cecal ligation and puncture; CORM-2, carbon monoxide releasing molecule-2; iCORM2, inactive CORM-2; i.v., intravenously.

difference was identified between the CLP group and the CLP+iCORM-2 group (Fig. 3).

Effect of CORM-2 on the expression of ICAM-1 and VCMP-1 in the pancreas of septic mice. The accumulation of neutrophils in the tissue results from the increased adherence of neutrophils to endothelial cells, which is mediated by adhesion molecules (26). Infiltration of neutrophils in the pancreas augments the injury of tissues. Therefore, the present study evaluated the expression levels of ICAM-1 and VCAM-1 adhesion molecules, which serve critical roles in the firm attachment of neutrophils to the endothelium. No positive staining for ICAM-1 was observed in the pancreatic tissue sections obtained from the sham group mice (Fig. 4A). Sections obtained from CLP-induced septic mice $24 \mathrm{~h}$ after CLP exhibited significant positive staining for ICAM-1 and VCAM-1 compared with the sham group $(\mathrm{P}<0.05$; Fig. 4A-C). The degree of pancreatic staining for ICAM-1 and VCAM-1 were significantly reduced in tissue sections obtained from $24 \mathrm{~h}$ post-CLP mice that had received CORM-2 treatment compared with the CLP-alone group ( $\mathrm{P}<0.05$; Fig. 4A-C). No significant changes in staining were observed in CLP-treated mice that had additionally been treated with iCORM-2 when compared with CLP-alone mice (Fig. 4A-C).

Effect of CORM-2 on the production of pro-inflammatory cytokines in the pancreas of septic mice. In order to investigate the regulation of inflammation in the pancreas by CORM-2 treatment, the expression levels of pro-inflammatory cytokines IL- 6 , IL- $1 \beta$ and TNF- $\alpha$ were determined using ELISA. The levels of IL-6, IL- $1 \beta$ and TNF- $\alpha$ significantly increased at 6 , 12 and $24 \mathrm{~h}$ after CLP when compared with the sham controls
$(\mathrm{P}<0.05$; Fig. 5A-C). Treatment with CORM-2 significantly decreased the CLP-induced increase in the expression of IL-6, IL-1 $\beta$ and TNF- $\alpha$ compared with the CLP-alone group $(\mathrm{P}<0.05$; Fig. 5A-C). Additionally, iCORM-2 treatment did not exert any effect in the expression of these cytokines (Fig. 5A-C).

Effect of CORM-2 on NF- $\kappa B$ activation in the pancreas of septic mice. To determine whether $\mathrm{NF}-\kappa \mathrm{B}$ was involved in the observed anti-inflammatory effects of CORM-2-derived $\mathrm{CO}$, the levels of $\mathrm{NF}-\kappa \mathrm{B}$ p65 in the pancreas were measured using western blot analysis. The results demonstrated that $24 \mathrm{~h}$ post-CLP mice exhibited a significantly increased protein level of $\mathrm{NF}-\kappa \mathrm{B}$ compared with the sham group mice $(\mathrm{P}<0.05$; Fig. 6A). This increase was significantly inhibited by treatment with CORM-2 compared with the CLP-alone group $(\mathrm{P}<0.05)$, whereas iCORM-2 treatment had no effect on the levels of

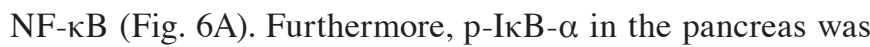
also examined using western blot analysis, which is required for the initiation of $N F-\kappa B$ activation. There was significant increase of the levels of $\mathrm{p}-\mathrm{I} \kappa \mathrm{B}-\alpha$ in the pancreas of septic mice $24 \mathrm{~h}$ after CLP compared with the sham group $(\mathrm{P}<0.05)$, which was significantly suppressed by the administration of CORM-2 compared with the CLP-alone group ( $\mathrm{P}<0.05$; Fig. 6B). No significant change in the $\mathrm{p}-\mathrm{I} \kappa \mathrm{B}-\alpha$ levels in the pancreas was observed by treatment with iCORM-2 when compared with the CLP-alone mice (Fig. 6B).

\section{Discussion}

Sepsis is a common phenomenon amongst critically ill patients, and is associated with the progressive failure of multiple organs (1). Although the most common dysfunctions of organs in sepsis are in the lung and kidney, the pancreas is also susceptible to inflammation and injury in patients with sepsis $(3,6)$. Research suggests that sepsis has a notable impact on pancreatic secretory function, which augments the severity of sepsis and is worse in septic shock compared with sepsis without shock (6). Impaired exocrine function in the pancreas is associated with Acute Physiology and Chronic Health Evaluation III and Sequential Organ Failure Assessment scores (6).

Previous studies have demonstrated the specific and independent function of exogenous $\mathrm{CO}$ in the modulation of inflammation (14,19,33-36). As novel metal carbonyl-based compounds, CORMs have the ability to deliver $\mathrm{CO}$ in biological systems in a controlled manner (11). CORM-derived $\mathrm{CO}$ has been demonstrated to have various benefits, including vasoactive, antihypertensive, cardio-protective and anti-rejection effects (37-40). CORM-2, one of the novel group of CORMs, has been determined to possess anti-inflammatory effects and to ameliorate the function of the pancreas in acute pancreatitis $(20,21)$. In previous studies, the administration of CORM-2 improved the function of numerous organs in CLP and thermal injury animal models of sepsis $(11,18)$. However, it remains unclear whether CORM-2 serves a protective effect on the function of the pancreas.

To determine the therapeutic function of $\mathrm{CO}$, the present study initially established CLP septic mice and sham mice. The animals were separated into four groups: Sham group; CLP group; CLP+CORM-2 group; and CLP+iCORM-2 
$\mathrm{A}_{\mathrm{a}}$

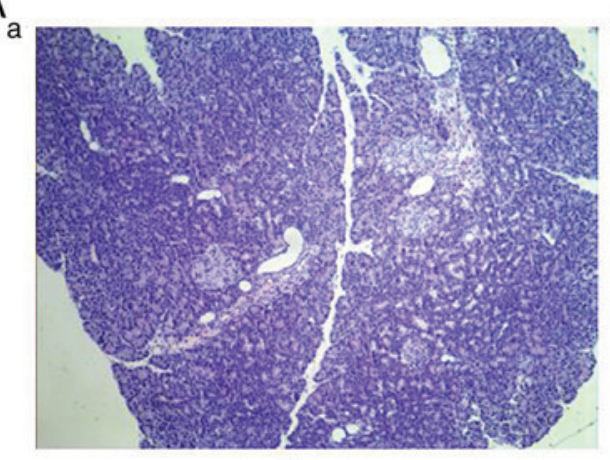

Sham

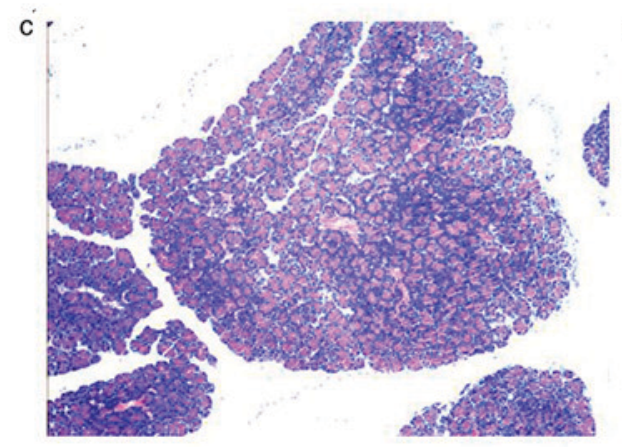

$\mathrm{CLP}+\mathrm{CORM}-2$

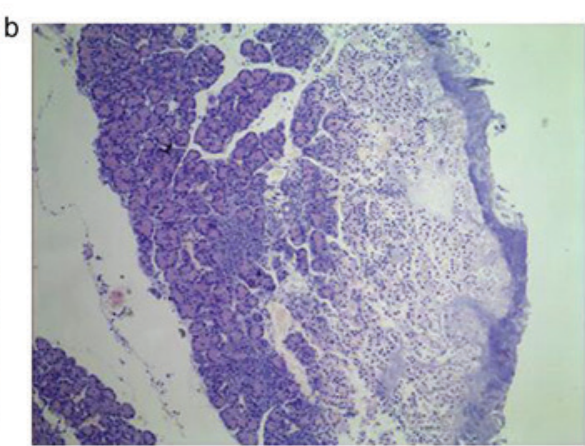

CLP

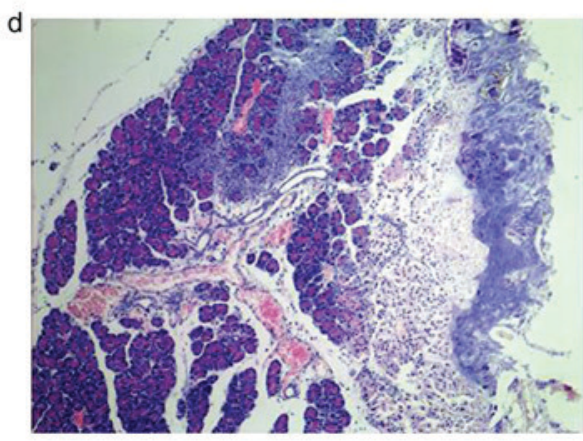

CLP+iCORM-2
B
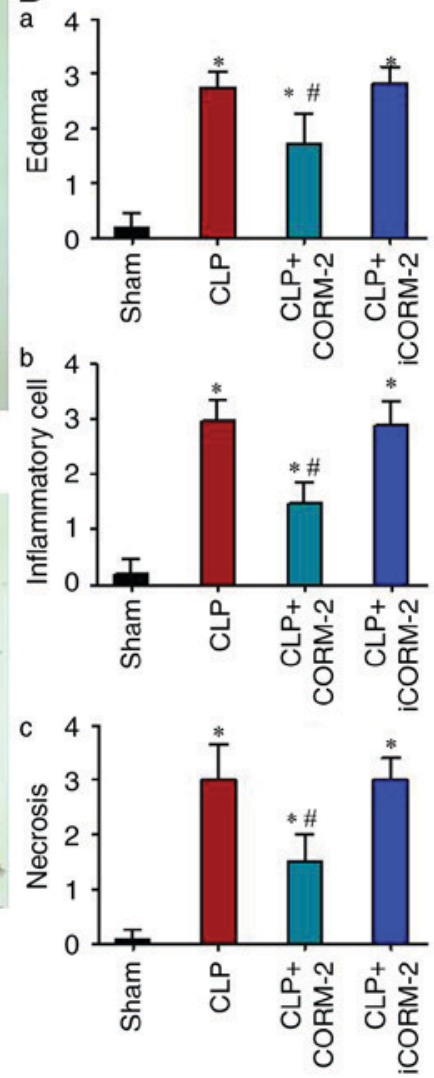

Figure 2. Histological evaluation of the effect of CLP and CORM-2 on the pancreas. (A) Effect of CORM-2 on pancreatic injury at $24 \mathrm{~h}$ after the induction of CLP (H\&E staining, magnification $\mathrm{x} 200$ ), in the pancreatic tissue obtained from: (Aa) The sham group, (Ab) the CLP group (mice subjected to CLP to induce polymicrobial sepsis), (Ac) the CLP+CORM-2 group (mice subjected to CLP and treated with CORM-2, 8 mg/kg, i.v.) and (Ad) the CLP+iCORM-2 group (mice subjected to CLP and treated with iCORM-2, $8 \mathrm{mg} / \mathrm{kg}$, i.v.). (B) Quantified effect of CORM-2 on histopathological scores of pancreatic injury. $\mathrm{H} \& \mathrm{E}$-stained sections were evaluated for $(\mathrm{Ba})$ edema, $(\mathrm{Bb})$ inflammatory cell infiltration and $(\mathrm{Bc})$ acinar necrosis. These data are expressed as the mean \pm standard deviation, $\mathrm{n}=10$ for each group. ${ }^{*} \mathrm{P}<0.05$ vs. the sham group; ${ }^{*} \mathrm{P}<0.05$ vs. the CLP group. CLP, cecal ligation and puncture; CORM-2, carbon monoxide releasing molecule-2; iCORM2, inactive CORM-2; i.v., intravenously; H\&E, hematoxylin and eosin.

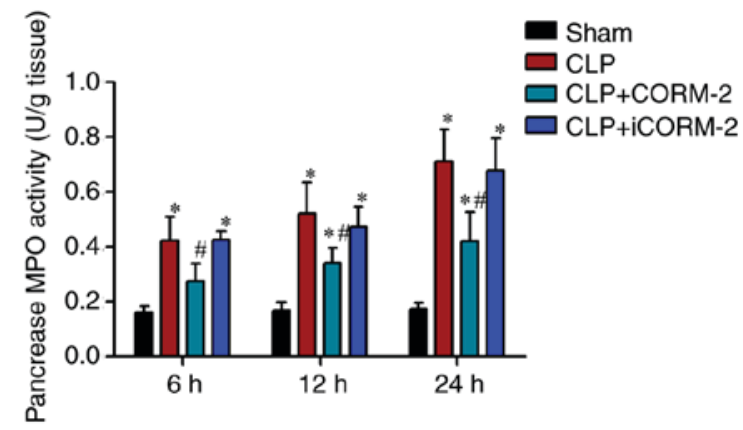

Figure 3. Effect of CORM-2 on the MPO activity in the pancreatic tissue of CLP-induced septic mice. MPO activity in the pancreas was measured at 6, 12 and $24 \mathrm{~h}$ after the induction of CLP in four groups: Sham, sham group; CLP, mice subjected to CLP to induce polymicrobial sepsis; CLP+CORM-2, mice subjected to CLP and treated with CORM-2 (8 mg/kg,i.v.); and CLP+iCORM-2, mice subjected to CLP and treated with iCORM-2 (8 mg/kg, i.v.). These data are expressed as the mean \pm standard deviation, $\mathrm{n}=5$ for each group. ${ }^{*} \mathrm{P}<0.05$ vs. the sham group; ${ }^{\#} \mathrm{P}<0.05$ vs. the CLP group. CLP, cecal ligation and puncture; CORM-2, carbon monoxide releasing molecule-2; iCORM2, inactive CORM-2; i.v., intravenously; MPO, myeloperoxidase.

group. Each group was divided again at postoperative 6, 12 and $24 \mathrm{~h}$ time points. CORM-2 or iCORM-2 were injected immediately following CLP. The levels of serum amylase and lipase (which are used to detect pancreatic injury) were examined in the present study. The results indicated that treatment with CORM-2 in CLP mice suppressed the levels of serum amylase and lipase compared with the sham group mice at all three time points. The septic mice treated with iCORM-2 did not exhibit altered serum amylase and lipase activity when compared with the CLP group mice at all three postoperative time points. Furthermore, the damage to the pancreas was also examined by histological evaluation. At all three time points, CORM-2 exerted a protective effect on the function of pancreas, and $24 \mathrm{~h}$ post-CLP exhibited the most substantial effect. Therefore, the present study selected the representative H\&E stained pancreatic sections in the four groups at the $24 \mathrm{~h}$ time point (Fig. 1). The pancreas from the mice of the sham group exhibited normal architecture, while the pancreatic tissues in the septic mice exhibited characteristic edema, inflammatory cell infiltration and necrosis of the acinar cells. Treatment with CORM-2 substantially reduced the extent and severity of the histological signs of pancreatic injury compared with the CLP-alone group, whereas alleviation in the injury of the pancreas was not observed in the iCORM-2 treatment group. The results were consistent with the experimental data of serum amylase and lipase levels. As the specific mechanisms for the therapeutic effects of 

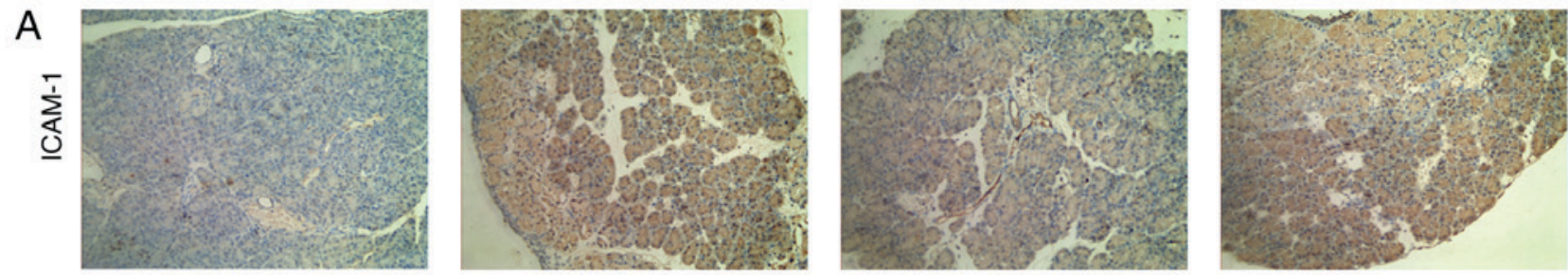

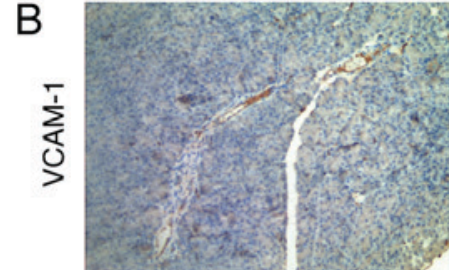

Sham

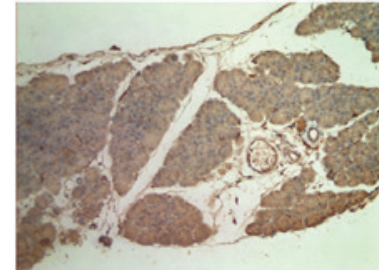

CLP

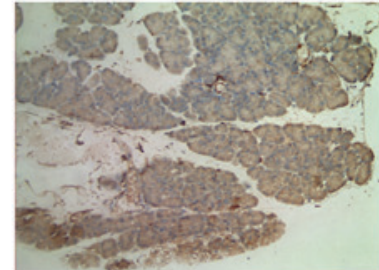

CLP+CORM-2

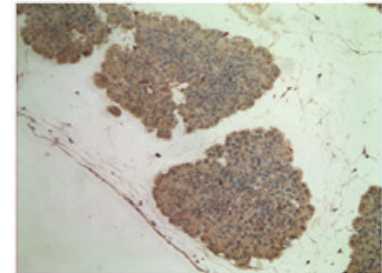

CLP+iCORM-2

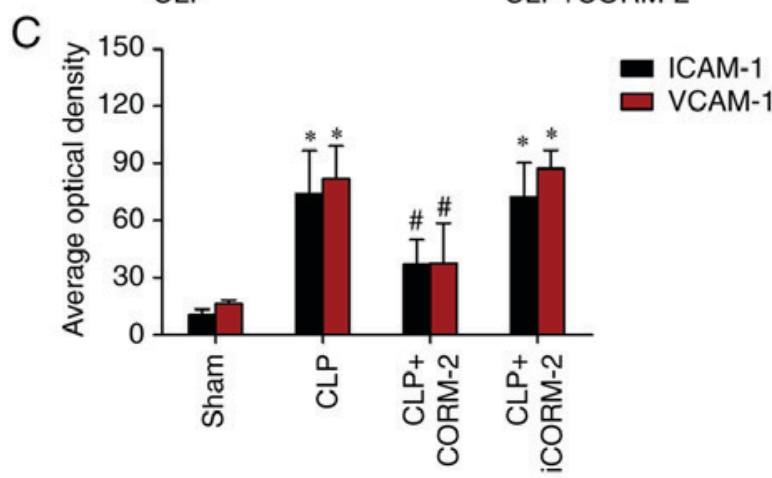

Figure 4. Effect of CORM-2 on ICAM-1 and VCAM-1 levels in the pancreatic tissue of CLP-induced septic mice (magnification, x200). Expression of (A) ICAM-1 and (B) VCAM-1 levels in the pancreas were determined at $24 \mathrm{~h}$ post-CLP by immunohistochemical staining, in the following four groups: Sham, sham group; CLP, mice subjected to CLP to induce polymicrobial sepsis; CLP+CORM-2, mice subjected to CLP and treated with CORM-2 (8 mg/kg, i.v.); and CLP+iCORM-2, mice subjected to CLP and treated with iCORM-2 (8 mg/kg, i.v.). (C) Quantitative analysis of the mean optical density for ICAM-1 and VCAM-1 in the pancreatic tissues. These data are expressed as the mean \pm standard deviation, $\mathrm{n}=10$ for each group. ${ }^{*} \mathrm{P}<0.05$ vs. the sham group; ${ }^{"} \mathrm{P}<0.05$ vs. the CLP group. CLP, cecal ligation and puncture; CORM-2, carbon monoxide releasing molecule-2; iCORM2, inactive CORM-2; i.v., intravenously; ICAM-1, intercellular adhesion molecule 1; VCAM-1, vascular cell adhesion molecule 1.

CORM-2 in CLP are unknown, the aim of the present study was to investigate the potential mechanisms and identify CORM-2 mediated cellular targets.

MPO is an enzyme that is present predominantly in the azurophilic granules of polymorphonuclears (PMNs), and is frequently used to estimate tissue PMN accumulation $(26,27)$. Neutrophil infiltration that aggravates tissue inflammation and damage is considered a principal contributor to sepsis mortality $(41,42)$. In the present study, it was revealed that MPO activity in the pancreas was substantially elevated at 6, 12 and $24 \mathrm{~h}$ after CLP compared with the sham group. These effects were notably attenuated following the administration of CORM-2 in vivo, but not iCORM-2, suggesting that CORM-2 effectively inhibited neutrophil chemotaxis and infiltration in the pancreas subsequent to CLP, eventually decreased the production of oxidants and reduced oxidant-mediated injury and the inflammatory response in the pancreas.

The direct cause of neutrophil infiltration into tissues following CLP is considered to be the overexpression of adhesion molecules (for example, ICAM-1 and VCAM-1) (36). Adhesion molecules activate neutrophils and endothelial cells, which in turn accelerate the release of various inflammatory mediators $(43,44)$. In the present study, immunohistochemical staining was used to detect the expression of ICAM-1 and VCAM-1 in pancreas tissue. The present results demonstrated that the expression levels of ICAM-1 and VCAM-1 in the pancreas were increased at $24 \mathrm{~h}$ post-CLP compared with the sham group. Administration of CORM-2 inhibited the upregulation of ICAM-1 and VCAM-induced by CLP. No significant change in the expression levels were observed in iCORM-2 treated CLP mice when compared with the CLP-alone treated mice.

Research has revealed that sepsis induces a widespread inflammatory response with a substantial increase of pro-inflammatory cytokines in the serum, which are important mediators in the pathogenesis of acute pancreatic injury (45). TNF- $\alpha$, IL-1 $\beta$ and IL-6 are induced in exaggerated production in the early stages of infectious diseases $(46,47)$. Studies have demonstrated that CORM-2 treatment abolishes the elevation in the levels of TNF- $\alpha$, IL-1 $\beta$ and IL- 6 in the serum in CLP-induced peritonitis sepsis and LPS-induced sterile sepsis $(3,20)$. Therefore, the levels of TNF- $\alpha$, IL-1 $\beta$ and IL- 6 in the pancreas were assessed in the present study. Similarly, the levels of TNF- $\alpha$, IL- $1 \beta$ and IL- 6 increased at 6, 12 and $24 \mathrm{~h}$ post-CLP compared with the sham group, which were attenuated by CORM-2 treatment. These data indicated that the cytoprotective effects of CORM-2 were, 


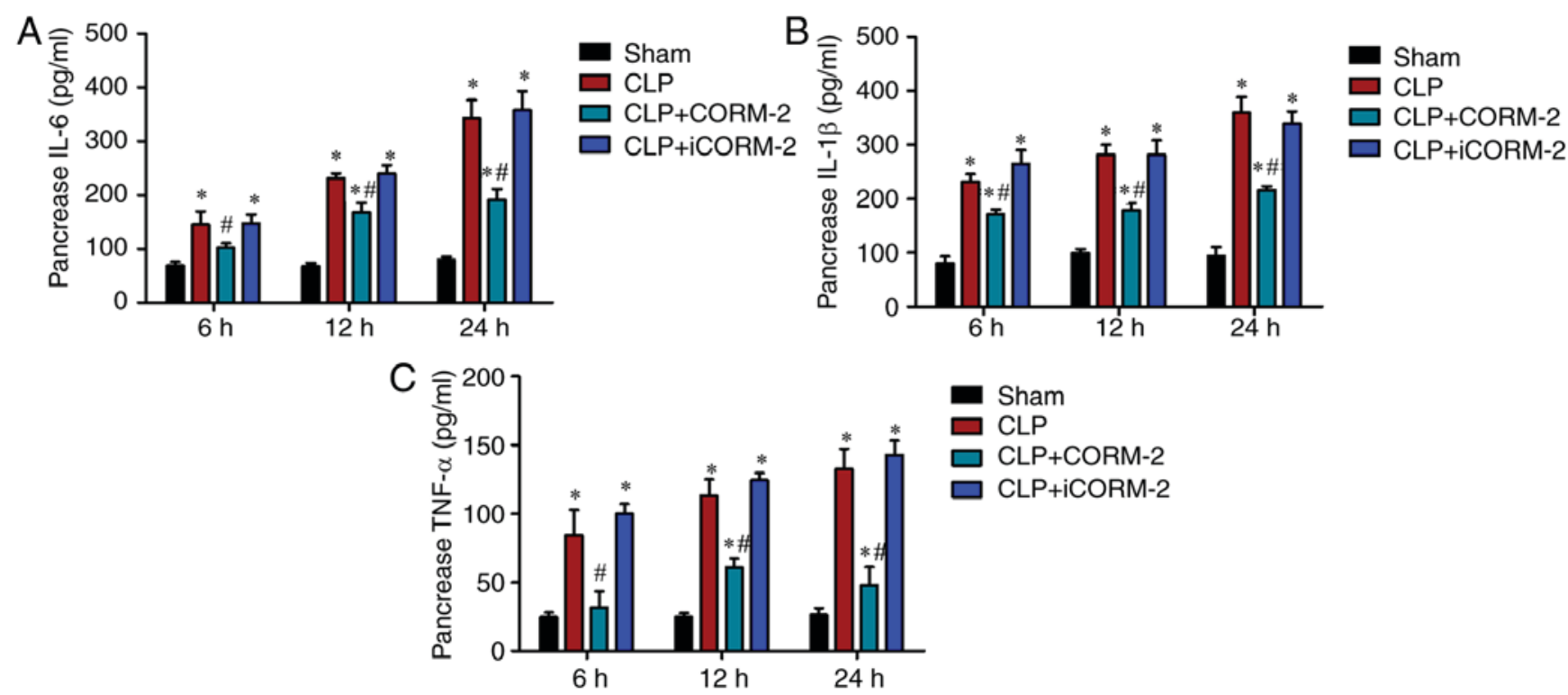

Figure 5. Effects of CORM-2 on the levels of pro-inflammatory cytokine in the pancreas of CLP-induced septic mice. The expression levels of (A) IL-6, (B) IL-1 $\beta$ and (C) TNF- $\alpha$ in the pancreas were assessed at 6,12 and $24 \mathrm{~h}$ after the induction of CLP or sham operation in the following four groups: Sham, sham group; CLP, mice subjected to CLP to induce polymicrobial sepsis; CLP+CORM-2, mice subjected to CLP and treated with CORM-2 (8 mg/kg, i.v.); CLP+iCORM-2, mice subjected to CLP and treated with iCORM-2 ( $8 \mathrm{mg} / \mathrm{kg}$, i.v.). These data are expressed as the mean \pm standard deviation, $\mathrm{n}=5$ for each group. ${ }^{*} \mathrm{P}<0.05$ vs. the sham group; ${ }^{*} \mathrm{P}<0.05$ vs. the CLP group. CLP, cecal ligation and puncture; CORM-2, carbon monoxide releasing molecule-2; iCORM2, inactive CORM-2; i.v., intravenously; IL-6, interleukin 6; IL-1 $\beta$, interleukin $1 \beta$; TNF- $\alpha$, tumor necrosis factor $\alpha$.

A
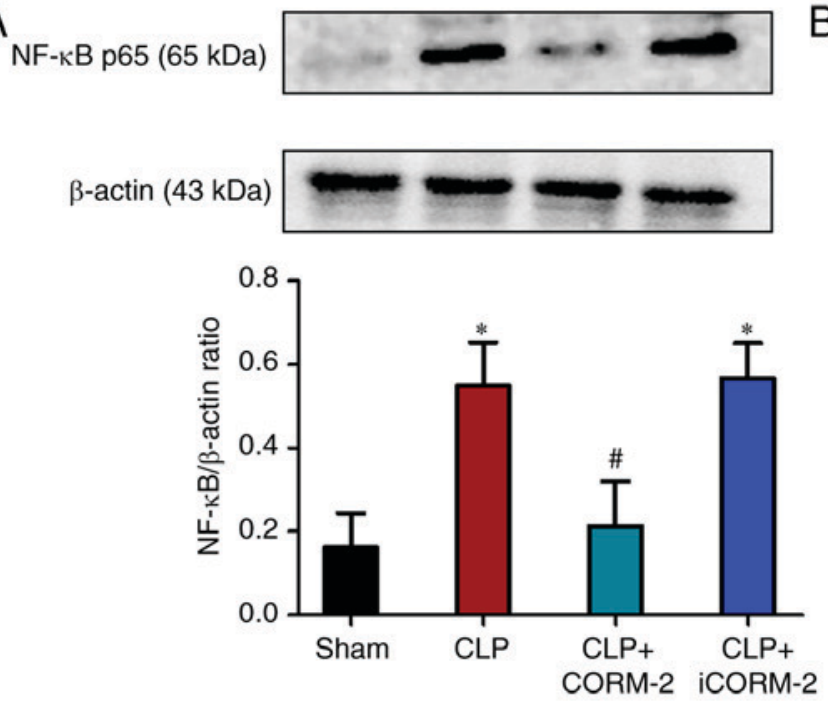

B
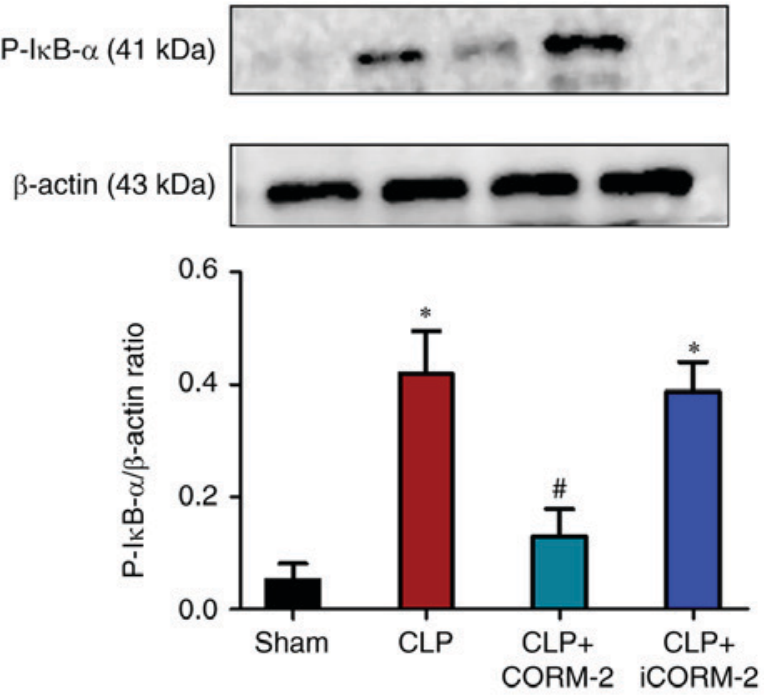

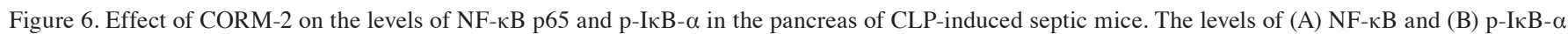
were analyzed using western blot analysis at $24 \mathrm{~h}$ after the induction of CLP or sham operation in the following four groups: Sham, sham group; CLP, mice subjected to CLP to induce polymicrobial sepsis; CLP+CORM-2, mice subjected to CLP and treated with CORM-2 (8 mg/kg, i.v.); and CLP+iCORM-2, mice subjected to CLP and treated with iCORM-2 ( $8 \mathrm{mg} / \mathrm{kg}$, i.v.). These data are expressed as the mean \pm standard deviation, $\mathrm{n}=5 \mathrm{for}$ each group. ${ }^{*} \mathrm{P}<0.05 \mathrm{vs}$. the sham group; ${ }^{~} \mathrm{P}<0.05$ vs. the CLP group. CLP, cecal ligation and puncture; CORM-2, carbon monoxide releasing molecule-2; iCORM2, inactive CORM-2; i.v., intravenously; NF- $\kappa \beta$, nuclear factor- $\kappa \beta$; $\mathrm{p}-\mathrm{I} \kappa \mathrm{B}-\alpha$, phosphorylated inhibitor of $\kappa \mathrm{B}$.

at least partly, due to the inhibition of the production and secretion of TNF- $\alpha$, IL-1 $\beta$ and IL-6 during the early period of sepsis.

The NF- $\mathrm{B}$ family members are ubiquitous, which rapidly trigger transcription factors that mediate immune and inflammatory reactions by regulating the expression of certain chemokines and cytokines $(48,49)$. Previously, it was reported that CORM-2 inhibited the activity of NF- $\mathrm{NB}$ in the tissues of the liver and lung in thermal injury mice models and E coli-induced murine sepsis models $(11,26)$. Therefore, the present study evaluated the involvement of $N F-\kappa B$ in the observed anti-inflammatory effects of CORM-2-derived $\mathrm{CO}$ on the pancreatic damage induced by CLP. For this purpose, the nuclear translocation and DNA binding of p65, the key component of NF- $\mathrm{B}$ (50-52), was measured in the pancreas using western blot analysis. Additionally, $\mathrm{p}-\mathrm{I} \kappa \mathrm{B}-\alpha$ in the pancreas was also examined using western blot analysis, which is required for the 
initiation of NF- $\kappa \mathrm{B}$ activation. The results indicated that

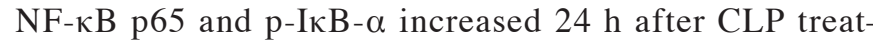
ment compared with the sham group, and this increase was suppressed by treatment with CORM-2. The levels of

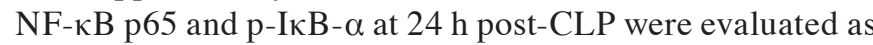
CORM-2 exerted a notable protective effect on the pancreatic function of septic mice $24 \mathrm{~h}$ post-CLP (as presented in Fig. 1). In fact, at post-operative 6 and $12 \mathrm{~h}$ time points,

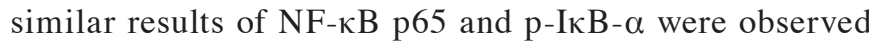
(data not shown). Inhibition of CORM-2 on the activation of NF- $\kappa \mathrm{B}$ p65 and p-IкB- $\alpha$ may explain the decrease in the expression of inflammatory factors including TNF- $\alpha$, IL- $1 \beta$ and IL- 6 observed in the pancreas. However, despite $\mathrm{p}-\mathrm{I} \kappa \mathrm{B}-\alpha$ being required for the initiation of NF- $\mathrm{KB}$ activation, and numerous other studies have only assessed the expression of $\mathrm{p}-\mathrm{I} \kappa \mathrm{B}-\alpha$ to demonstrate the activation of NF- $\mathrm{BB}$ (53-55), in order for the experimental results to be more convincing, total IкB- $\alpha$ also needs to be tested. This is a limitation of the present study.

Despite the therapeutic and anti-inflammatory effects of exogenous $\mathrm{CO}$ in various disease and injury models, there has been little progress with regard to its use in clinical illness. The main problem is the method of administration of $\mathrm{CO}$. Although low dose inhalational $\mathrm{CO}$ has been revealed to have anti-inflammatory effects against ventilatory-induced lung injury and has been associated with a decreased level of TNF- $\alpha(21,56)$, it is difficult to ensure a certain dose range of therapeutic $\mathrm{CO}$ without increasing the level of CO-hemoglobin (57). CORM-2 or iCORM-2, at the concentrations used in the present study, have previously been demonstrated not to be toxic to mammalian cells in vitro and to mice in vivo $(21,58)$. However, the safety of intravenous injection with CORM-2 in vivo has not been demonstrated satisfactorily. Therefore, a novel method of administration of $\mathrm{CO}$ requires further investigation.

In summary, the present study demonstrated that the application of CORM-2 attenuated the severity of sepsis and improved the function of the pancreas. The mechanism by which CORM-2-derived CO inhibited pro-inflammatory cytokines of the pancreas may be via inhibiting NF- $\mathrm{KB}$ activation. However, this was a preliminary study on the function of CORM-2 in pancreatic function in sepsis model mice and its mechanism of action. There remain numerous issues that require further investigation, for example, whether the effect of CORM-2 on NF- $\mathrm{KB}$ activation is direct or indirect; what will happen if NF- $\mathrm{BB}$ and/or $\mathrm{p}-\mathrm{I} \kappa \mathrm{B}-\alpha$ are blocked; and if NF- $\kappa \mathrm{B}$ and/or P-I $\kappa \mathrm{B}-\alpha$ are blocked, will any reverse effect occur or not. Future studies will conduct in-depth research on these issues.

\section{Acknowledgements}

Not applicable.

\section{Funding}

The present study was supported by the National Natural Science Foundation of China (grant nos. 81272148 and 81471903) and the Jiangsu Natural Science Foundation (grant no. BK2012703).

\section{Availability of data and materials}

The analyzed data sets generated during the present study are available from the corresponding author on reasonable request.

\section{Authors' contributions}

BS designed the study. YL was responsible for data access and analysis and manuscript preparation. $\mathrm{XW}, \mathrm{XX}$ and WQ collaborated to perform data analysis and interpret the results.

\section{Ethics approval and consent to participate}

The present study was approved by the Ethics Committee of Affiliated Hospital of Jiangsu University (Jiangsu, China).

\section{Patient consent for publication}

Not applicable.

\section{Competing interests}

The authors declare that they have no competing interests.

\section{References}

1. Abou Dagher G, Harmouche E, Jabbour E, Bachir R, Zebian D and Bou Chebl R: Sepsis in hemodialysis patients. BMC Emerg Med 15: 30, 2015.

2. Aikawa N: Revised Surviving Sepsis Campaign Guidelines and therapy for severe sepsis. Jpn J Antibiot 64: 37-44, 2011 (In Japanese).

3. Liu Z, Shi Q, Liu J, Abdel-Razek O, Xu Y, Cooney RN and Wang G: Innate immune molecule surfactant protein $\mathrm{D}$ attenuates sepsis-induced acute pancreatic injury through modulating apoptosis and NF-kB-mediated inflammation. Sci Rep 5: 17798, 2015.

4. Levy MM, Fink MP, Marshall JC, Abraham E, Angus D, Cook D, Cohen J, Opal SM, Vincent JL and Ramsay G; SCCM/ESICM/ACCP/ATS/SIS: 2001 SCCM/ESICM/ACCP/ATS/SIS International Sepsis Definitions Conference. Crit Care Med 31: 1250-1256, 2003.

5. Marshall JC, Cook DJ, Christou NV, Bernard GR, Sprung CL and Sibbald WJ: Multiple organ dysfunction score: A reliable descriptor of a complex clinical outcome. Crit Care Med 23: 1638-1652, 1995.

6. Tribl B, Sibbald WJ, Vogelsang H, Spitzauer S, Gangl A and Madl C: Exocrine pancreatic dysfunction in sepsis. Eur J Clin Invest 33: 239-243, 2003.

7. Tribl B, Madl C, Mazal PR, Schneider B, Spitzauer S, Vogelsang $\mathrm{H}$ and Gangl A: Exocrine pancreatic function in critically ill patients: Septic shock versus non-septic patients. Crit Care Med 28: 1393-1398, 2000.

8. Sun BW and Chen X: Carbon monoxide releasing molecules: New insights for anticoagulation strategy in sepsis. Cell Mol Life Sci 66: 365-369, 2009.

9. Soni H, Pandya G, Patel P, Acharya A, Jain M and Mehta AA: Beneficial effects of carbon monoxide-releasing molecule-2 (CORM-2) on acute doxorubicin cardiotoxicity in mice: Role of oxidative stress and apoptosis. Toxicol Appl Pharmacol 253: 70-80, 2011.

10. Chung SW, Liu X, Macias AA, Baron RM and Perrella MA: Heme oxygenase-1-derived carbon monoxide enhances the host defense response to microbial sepsis in mice. J Clin Invest 118: 239-247, 2008.

11. Sun B, Sun Z, Jin Q and Chen X: CO-releasing molecules (CORM-2)-liberated $\mathrm{CO}$ attenuates leukocytes infiltration in the renal tissue of thermally injured mice. Int J Biol Sci 4: 176-183, 2008. 
12. Lee S, Lee SJ, Coronata AA, Fredenburgh LE, Chung SW, Perrella MA, Nakahira K, Ryter SW and Choi AM: Carbon monoxide confers protection in sepsis by enhancing beclin 1-dependent autophagy and phagocytosis. Antioxid Redox Signal 20: 432-442, 2014.

13. Coburn RF: The measurement of endogenous carbon monoxide production. J Appl Physiol (1985) 112: 1949-1955, 2012.

14. Ryter SW and Choi AM: Carbon monoxide: Present and future indications for a medical gas. Korean J Intern Med 28: 123-140, 2013.

15. Ozaki KS, Yoshida J, Ueki S, Pettigrew GL, Ghonem N, Sico RM, Lee LY, Shapiro R, Lakkis FG, Pacheco-Silva A and Murase N: Carbon monoxide inhibits apoptosis during cold storage and protects kidney grafts donated after cardiac death. Transpl Int 25: 107-117, 2012

16. Liu DM, Sun BW, Sun ZW, Jin Q, Sun Y and Chen X Suppression of inflammatory cytokine production and oxidative stress by $\mathrm{CO}$-releasing molecules-liberated $\mathrm{CO}$ in the small intestine of thermally-injured mice. Acta Pharmacol Sin 29: 838-846, 2008.

17. Katada K, Bihari A, Mizuguchi S, Yoshida N, Yoshikawa T, Fraser DD, Potter RF and Cepinskas G: Carbon monoxide liberated from CO-releasing molecule (CORM-2) attenuates ischemia/reperfusion (I/R)-induced inflammation in the small intestine. Inflammation 33: 92-100, 2010.

18. Sun BW, Jin Q, Sun Y, Sun ZW, Chen X, Chen ZY and Cepinskas G: Carbon liberated from CO-releasing molecules attenuates leukocyte infiltration in the small intestine of thermally injured mice. World J Gastroenterol 13: 6183-6190, 2007.

19. Sun B, Sun H, Liu C, Shen J, Chen Z and Chen X: Role of $\mathrm{CO}$-releasing molecules liberated $\mathrm{CO}$ in attenuating leukocytes sequestration and inflammatory responses in the lung of thermally injured mice. J Surg Res 139: 128-135, 2007.

20. Chen P, Sun B, Chen H, Wang G, Pan S, Kong R, Bai X and Wang S: Effects of carbon monoxide releasing molecule-liberated $\mathrm{CO}$ on severe acute pancreatitis in rats. Cytokine 49: 15-23, 2010

21. Xue J and Habtezion A: Carbon monoxide-based therapy ameliorates acute pancreatitis via TLR4 inhibition. J Clin Invest 124 437-447, 2014

22. Liu D, Liang F, Wang X, Cao J, Qin W and Sun B: Suppressive effect of CORM-2 on LPS-induced platelet activation by glycoprotein mediated HS1 phosphorylation interference. PLoS One 8: e83112, 2013

23. Liu J, Abdel-Razek O, Liu Z, Hu F, Zhou Q, Cooney RN and Wang G: Role of surfactant proteins A and D in sepsis-induced acute kidney injury. Shock 43: 31-38, 2015.

24. Tang Z, Ni L, Javidiparsijani S, Hu F, Gatto LA, Cooney R and Wang G: Enhanced liver autophagic activity improves survival of septic mice lacking surfactant proteins A and D. Tohoku J Exp Med 231: 127-138, 2013.

25. Deng W, Hui Y, Yu J, Wang W, Xu S, Chen C and Xiong X: A new pathological scoring method for adrenal injury in rats with severe acute pancreatitis. Pathol Res Pract 210: 1011-1017, 2014.

26. Shen WC, Wang X, Qin WT, Qiu XF and Sun BW: Exogenous carbon monoxide suppresses Escherichia coli vitality and improves survival in an Escherichia coli-induced murine sepsis model. Acta Pharmacol Sin 35: 1566-1576, 2014

27. Hillegass LM, Griswold DE, Brickson B and Albrightson-Winslow C: Assessment of myeloperoxidase activity in whole rat kidney. J Pharmacol Methods 24: 285-295, 1990.

28. Zhou X, Liu Z, Jang F, Xiang C, Li Y and He Y: Autocrine sonic hedgehog attenuates inflammation in cerulein-induced acute pancreatitis in mice via upregulation of IL-10. PLoS One 7: e44121, 2012

29. Tribl B, Bateman RM, Milkovich S, Sibbald WJ and Ellis CG: Effect of nitric oxide on capillary hemodynamics and cell injury in the pancreas during Pseudomonas pneumonia-induced sepsis. Am J Physiol Heart Circ Physiol 286: H340-H345, 2004

30. Pieracci FM and Barie PS: Management of severe sepsis of abdominal origin. Scand J Surg 96: 184-196, 2007.

31. Kong XY, Du YQ, Li L, Liu JQ, Wang GK, Zhu JQ, Man XH, Gong YF, Xiao LN, Zheng YZ, et al: Plasma miR-216a as a potential marker of pancreatic injury in a rat model of acute pancreatitis. World J Gastroenterol 16: 4599-4604, 2010.

32. Choudhury S, Kandasamy K, Maruti BS, Addison MP, Kasa JK, Darzi SA, Singh TU, Parida S, Dash JR, Singh V and Mishra SK: Atorvastatin along with imipenem attenuates acute lung injury in sepsis through decrease in inflammatory mediators and bacterial load. Eur J Pharmacol 765: 447-456, 2015.
33. Queiroga CS, Vercelli A and Vieira HL: Carbon monoxide and the CNS: Challenges and achievements. Br J Pharmacol 172: $1533-1545,2015$

34. Song M, Wang X, Qin W, Zhuang M, Xu X, Zhang Y and Sun B: Effects of exogenous carbon monoxide-releasing molecule 2 intervention in vitro on formation of human neutrophil extracellular traps stimulated by endotoxin/lipopolysaccharide and its mechanism. Zhonghua Shao Shang Za Zhi 32: 82-88, 2016 (In Chinese).

35. Liu D, Wang X, Qin W, Chen J, Wang Y, Zhuang M and Sun B: Suppressive effect of exogenous carbon monoxide on endotoxin-stimulated platelet over-activation via the glycoprotein-mediated PI3K-Akt-GSK3 $\beta$ pathway. Sci Rep 6: 23653 , 2016.

36. Qin W, Zhang J, Lv W, Wang X and Sun B: Effect of carbon monoxide-releasing molecules II-liberated $\mathrm{CO}$ on suppressing inflammatory response in sepsis by interfering with nuclear factor kappa B activation. PLoS One 8: e75840, 2013.

37. Motterlini R, Clark JE, Foresti R, Sarathchandra P, Mann BE and Green CJ: Carbon monoxide-releasing molecules: Characterization of biochemical and vascular activities. Circ Res 90: E17-E24, 2002.

38. Motterlini R, Sawle P, Hammad J, Bains S, Alberto R, Foresti R and Green CJ: CORM-A1: A new pharmacologically active carbon monoxide-releasing molecule. FASEB J 19: 284-286, 2005.

39. Johnson TR, Mann BE, Clark JE, Foresti R, Green CJ and Motterlini R: Metal carbonyls: A new class of pharmaceuticals? Angew Chem Int Ed Engl 42: 3722-3729, 2005.

40. Clark JE, Naughton P, Shurey S, Green CJ, Johnson TR, Mann BE, Foresti R and Motterlini R: Cardioprotective actions by a water-soluble carbon monoxide-releasing molecule. Circ Res 93: e2-e8, 2003.

41. Cioffi WG, Burleson DG and Pruitt BA Jr: Leukocyte responses to injury. Arch Surg 128: 1260-1267, 1993.

42. Bradley PP, Priebat DA, Christensen RD and Rothstein G: Measurement of cutaneous inflammation: Estimation of neutrophil content with an enzyme marker. J Invest Dermatol 78: 206-209, 1982

43. Christofidou-Solomidou M, Nakada MT, Williams J, Muller WA and DeLisser HM: Neutrophil platelet endothelial cell adhesion molecule-1 participates in neutrophil recruitment at inflammatory sites and is down-regulated after leukocyte extravasation. J Immunol 158: 4872-4878, 1997.

44. Imamoto E, Yoshida N, Uchiyama K, Kuroda M, Kokura S, Ichikawa H, Naito Y, Tanigawa T and Yoshikawa T: Inhibitory effect of pioglitazone on expression of adhesion molecules on neutrophils and endothelial cells. Biofactors 20: 37-47, 2004.

45. Pereda J, Sabater L, Aparisi L, Escobar J, Sandoval J, Viña J, López-Rodas G and Sastre J: Interaction between cytokines and oxidative stress in acute pancreatitis. Curr Med Chem 13: 2775-2787, 2006

46. Kim GY, Roh SI, Park SK, Ahn SC, Oh YH, Lee JD and Park YM: Alleviation of experimental septic shock in mice by acidic polysaccharide isolated from the medicinal mushroom Phellinus linteus. Biol Pharm Bull 26: 1418-1423, 2003.

47. Xiang K, Cheng L, Luo Z, Ren J, Tian F, Tang L, Chen T and Dai R: Glycyrrhizin suppresses the expressions of HMGB1 and relieves the severity of traumatic pancreatitis in rats. PLoS One 9: e115982, 2014

48. Baeuerle PA and Henkel T: Function and activation of NF-kappa $\mathrm{B}$ in the immune system. Annu Rev Immunol 12: 141-179, 1994.

49. Yin MJ, Yamamoto $Y$ and Gaynor RB: The anti-inflammatory agents aspirin and salicylate inhibit the activity of I(kappa)B kinase-beta. Nature 396: 77-80, 1998.

50. Han B, Ji B and Logsdon CD: CCK independently activates intracellular trypsinogen and NF-kappaB in rat pancreatic acinar cells. Am J Physiol Cell Physiol 280: C465-C472, 2001.

51. Kulms D and Schwarz T: NF-kappaB and cytokines. Vitam Horm 74: 283-300, 2006

52. Song YS, Lee YS, Narasimhan P and Chan PH: Reduced oxidative stress promotes NF-kappaB-mediated neuroprotective gene expression after transient focal cerebral ischemia: Lymphocytotrophic cytokines and antiapoptotic factors. J Cereb Blood Flow Metab 27: 764-775, 2007.

53. Dai B, Lei C, Lin R, Tao L, Bin Y, Peng H and Lei B: Activation of liver $X$ receptor $\alpha$ protects amyloid $\beta_{1-40}$ induced inflammatory and senescent responses in human retinal pigment epithelial cells. Inflamm Res 66: 523-534, 2017. 
54. Tao L, Qiu Y, Fu X, Lin R, Lei C, Wang J and Lei B: Angiotensin-converting enzyme 2 activator diminazene aceturate prevents lipopolysaccharide-induced inflammation by inhibiting MAPK and NF- $\kappa$ B pathways in human retinal pigment epithelium. J Neuroinflammation 13: 35, 2016.

55. Lu Y, Li F, Xu T and Sun J: Tetrandrine prevents multidrug resistance in the osteosarcoma cell line, U-2OS, by preventing Pgp overexpression through the inhibition of NF- $\mathrm{KB}$ signaling. Int J Mol Med 39: 993-1000, 2017.

56. Dolinay T, Szilasi M, Liu M and Choi AM: Inhaled carbon monoxide confers antiinflammatory effects against ventilator-induced lung injury. Am J Respir Crit Care Med 170: 613-620, 2004.
57. El-Mousleh T, Casalis PA, Wollenberg I, Zenclussen ML, Volk HD, Langwisch S, Jensen F and Zenclussen AC: Exploring the potential of low doses carbon monoxide as therapy in pregnancy complications. Med Gas Res 2: 4, 2012.

58. Desmard M, Foresti R, Morin D, Dagouassat M, Berdeaux A, Denamur E, Crook SH, Mann BE, Scapens D, Montravers P, et al: Differential antibacterial activity against Pseudomonas aeruginosa by carbon monoxide-releasing molecules. Antioxid Redox Signal 16: 153-163, 2012.

(i) (9) This work is licensed under a Creative Commons EY No No Attribution-NonCommercial-NoDerivatives 4.0 International (CC BY-NC-ND 4.0) License. 